\title{
Misattributed parentage as an unanticipated finding during exome/genome sequencing: current clinical laboratory practices and an opportunity for standardization
}

\author{
Celeste Eno, $\mathrm{PhD}^{1}$, Pinar Bayrak-Toydemir, MD, PhD², Lora Bean, PhD, FACMG ${ }^{3,4}$, \\ Alicia Braxton, MS, CGC ${ }^{5}$, Elizabeth C. Chao, MD, FACMG ${ }^{6}$, Dima El-Khechen, MS, CGC \\ Edward D. Esplin, MD, PhD ${ }^{8}$, Bethany Friedman, MS, $\mathrm{CGC}^{9}$, Kelly D. Farwell Hagman, MS, $\mathrm{CGC}^{7}$, \\ Tina Hambuch, PhD, FACMG ${ }^{8}$, Amy Hernandez, MS, CGC ${ }^{10}$, Jane Juusola, PhD, FACMG ${ }^{9}$, \\ Gina Londre, MS, $\mathrm{CGC}^{11}$, Jerry Machado, PhD, FACMG ${ }^{11}$, Rong Mao, MD, FACMG ${ }^{2}$, \\ Lindsey Mighion, MS, $\mathrm{CGC}^{3}$, Heidi L. Rehm, PhD, FACMG ${ }^{10}$, Patricia Ward, MS, CGC ${ }^{5}$ and \\ Joshua L. Deignan, PhD, FACMG ${ }^{1}$
}

\begin{abstract}
Purpose: Clinical laboratories performing exome or genome sequencing $(\mathrm{ES} / \mathrm{GS})$ are familiar with the challenges associated with proper consenting for and reporting of medically actionable secondary findings based on recommendations from the American College of Medical Genetics and Genomics (ACMG). Misattributed parentage is another type of unanticipated finding a laboratory may encounter during family-based ES/GS; however, there are currently no professional recommendations related to the proper consenting for and reporting of misattributed parentage encountered during ES/GS.
\end{abstract}

Methods: We surveyed 10 clinical laboratories offering familybased ES/GS regarding their consent language, discovery, and reporting of misattributed parentage.

Results: Many laboratories have already developed their own practices/policies for these issues, which do not necessarily agree with those from other labs.
Conclusion: There are several other possibilities besides true misattributed parentage that could result in similar laboratory findings, and laboratories often feel they lack sufficient information to make formal conclusions on a report regarding the true genetic relatedness of the submitted samples. However, understanding the genetic relatedness (or lack thereof) of the samples submitted for family-based ES/GS has medical relevance. Therefore, professional recommendations for the appropriate handling of suspected misattributed parentage encountered during ES/GS are needed to help standardize current clinical laboratory practices.

Genetics in Medicine (2019) 21:861-866; https://doi.org/10.1038/s41436018-0265-4

Keywords: exome; genome; sequencing; parentage; ethics

\section{INTRODUCTION}

Clinical genomic testing using next-generation sequencing (NGS) has greatly expanded over the past decade due to a decrease in test costs and an increased interest in the capabilities of these methods by patients, clinicians, and laboratory personnel. Exome and genome sequencing now allow for the rapid identification of causal variants for previously undiagnosed conditions. However, the ability to sequence the exome or genome results in additional challenges, which clinical laboratories are then required to address. One such issue was how to consent for and report any medically actionable secondary findings, which now have published recommendations from the American College of Medical Genetics and Genomics (ACMG). ${ }^{1,2}$ Another issue was how to document suspected consanguinity. ${ }^{3,4}$ However, one issue where the ACMG does not currently have any published recommendations to assist laboratories is how to properly consent for and report (or not report) misattributed parentage encountered during family-based exome or genome sequencing (trio/duo ES/GS). Thus, current consent language and practices for handling potential evidence of misattributed parentage vary significantly between clinical laboratories.

\footnotetext{
${ }^{1}$ University of California, Los Angeles, Los Angeles, CA, USA; ${ }^{2}$ ARUP Laboratories, University of Utah, Salt Lake City, UT, USA; ${ }^{3}$ EGL Genetics, Tucker, GA, USA; ${ }^{4}$ Emory University, Atlanta, GA, USA; ${ }^{5}$ Baylor Genetics, Baylor College of Medicine, Houston, TX, USA; ${ }^{6}$ University of California, Irvine, Irvine, CA, USA; ${ }^{7}$ Ambry Genetics, Aliso Viejo, CA, USA; ${ }^{8}$ Invitae Corporation, San Francisco, CA, USA; ${ }^{9}$ GeneDx, Gaithersburg, MD, USA; ${ }^{10}$ Partners HealthCare, Laboratory for Molecular Medicine, Cambridge, MA, USA; ${ }^{11}$ PreventionGenetics, Marshfield, WI, USA. Correspondence: Joshua L. Deignan (JDeignan@mednet.ucla.edu)
} 

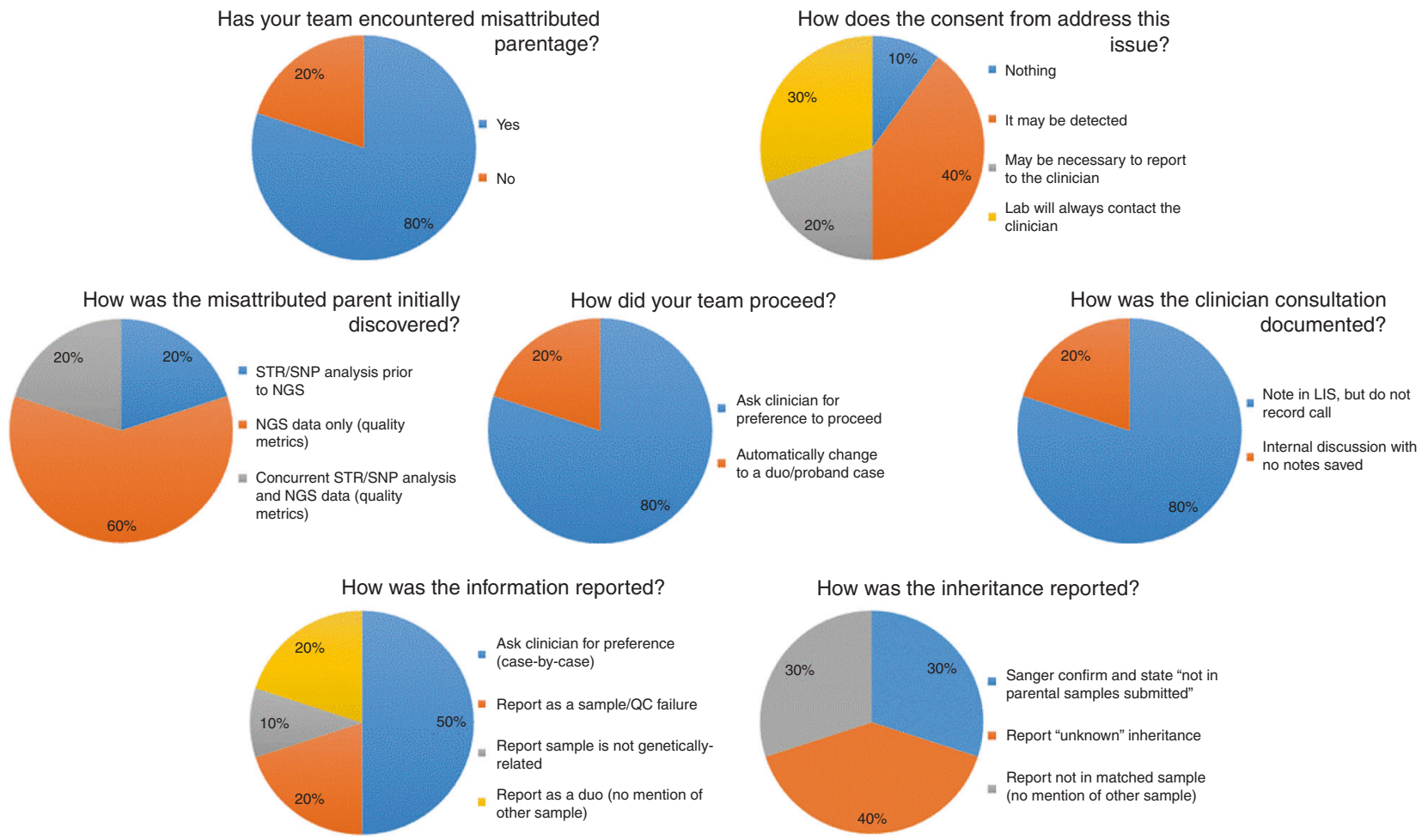

Fig. 1 Results from a laboratory survey on misattributed parentage in exome/genome sequencing (ES/GS). All laboratories surveyed indicate that they offer trio sequencing. LIS laboratory information system, NGS next-generation sequencing, QC quality control, SNP single-nucleotide polymorphism, STR short tandem repeat

Disclosure of misattributed parentage encountered during clinical laboratory testing has always been controversial. While the President's Commission for the Study of Ethical Problems in Biomedical and Behavioral Research ${ }^{5}$ recommended disclosure of misattributed parentage to both partners, the Committee on Assessing Genetic Risks of the Institute of Medicine (IOM) recommended disclosure to the mother and not to the assumed father based on the rationale that "genetic testing should not be used in ways that disrupt families." ${ }^{\prime \prime}$ An additional study reported that $44 \%$ and $39 \%$ of geneticists surveyed would prefer to exclude this information from laboratory reports for pediatric and adult cases, respectively, ${ }^{7}$ and when they accounted for the respondents' profession, they found significantly more laboratory and clinical geneticists compared with genetic counselors would prefer to exclude this information. The American Society of Human Genetics (ASHG) also currently recommends avoiding disclosure unless there is a medical benefit. ${ }^{8}$

While this study focuses solely on the practices of disclosure by clinical laboratories and not the practices of disclosure by clinicians, clinicians will frequently use the laboratory report as a guide for discussion with the patient and his/her family on this topic. Because many patients may also ask for a hard copy of their laboratory report, it becomes challenging for the clinician to potentially withhold any information contained in the report. The laboratory report may also be entered into the electronic medical record, and thus, any future health-care professional may disclose the information to the family even if the original ordering clinician chose not to do so.

\section{MATERIALS AND METHODS}

We identified and surveyed a total of ten clinical laboratories (three academic, seven commercial) currently offering familybased ES/GS using both phone interviews and written questionnaires regarding their current practices/policies and consent language used for cases with suspected misattributed parentage (focusing mainly on misattributed paternity). Only ten clinical laboratories were approached, and all ten agreed to participate in this study. We asked individuals from each laboratory (one to two individuals per laboratory, 11 laboratorians and eight genetic counselors in total) if they have previously encountered misattributed parentage, how it was initially discovered, and how their institution/laboratory typically handles these situations. In total, the initial phone interviews were between 30 and 60 minutes in length. Initial interview responses were documented, evaluated, and used to develop a structured questionnaire (Sup. Fig. 1). The questionnaire with multiple choice answers was sent to the respective institutions for confirmation of their responses. All answers were then collected and evaluated. For the two laboratories that had not yet encountered misattributed parentage at the time of the survey, they were asked to respond to the remaining questions ( 3 through 8 ) based on how they would likely handle these issues if they were to encounter misattributed parentage in the future. 


\section{RESULTS}

All of the laboratories surveyed indicate that they offer trio sequencing. On their ES/GS consent form, the majority of laboratories $(90 \%)$ inform patients that misattributed parentage may be detected, with $50 \%$ of the laboratories indicating that the ordering clinician may (or will) be informed about it if it is discovered (Fig. 1). The most explicit consent form among the surveyed laboratories included a section stating that misattributed parentage uncovered during testing will result in notification to the clinician and cancellation of the test. However, that laboratory recently changed its consent form to remove this statement; it was not preventing samples with suspected misattributed parentage from being sent to their laboratory, and clients often wanted the option to continue the test by either submitting a new parental sample or changing the request to a duo instead of having the laboratory immediately cancel the order.

Most (60\%) of the laboratories surveyed proceed directly to ES/GS and rely on internal NGS quality metrics to assess the genetic relatedness of the samples (with or without downstream short tandem repeat [STR] analysis for confirmation). The remaining laboratories (40\%) surveyed utilize upfront (before NGS) or concurrent (in parallel with NGS) STR analysis to confirm the genetic relatedness of the samples.

All of the laboratories surveyed inform the ordering clinician when misattributed parentage is encountered. Eighty percent of the laboratories document the details of that discussion with the clinician in their laboratory information system; $20 \%$ only have verbal or electronic discussions with the ordering clinician about the results without documentation in their laboratory information system. If misattributed parentage is suspected, $80 \%$ of the laboratories ask the ordering clinician for their preference for either changing the test mode (from a trio to a duo or a proband-only analysis), canceling the order, or asking for a sample from a different genetically related individual (such as an unaffected sibling) instead to complete the analysis. Only $10 \%$ of the laboratories indicated that they routinely ask for a new blood sample from the mismatched parent to eliminate the possibility of a specimen mix-up (which is then assessed via concordance of STR results prior to resequencing); however, this is usually only pursued after an internal laboratory mix-up has been ruled out through reextraction of the remnant blood sample.

Fifty percent of the laboratories routinely abide by the reporting preferences of the ordering clinician, resulting in a variety of reporting practices within an individual laboratory that occur on a case-by-case basis. Another $20 \%$ of the laboratories automatically issue the final report as a duo using only the genetically related parent for the analysis without mentioning suspected misattributed parentage in the report. Twenty percent of the laboratories report these findings as a sample/quality control (QC) failure, and $10 \%$ of the laboratories surveyed specifically report that the submitted sample does not appear to be genetically related to the proband after phlebotomy and laboratory mix-ups have both been ruled out. None of the laboratories surveyed would report a variant as de novo if one of the submitted parental samples did not appear to be genetically related to the proband; they would instead state the inheritance using language such as "unknown" or "not in the parental samples submitted."

\section{DISCUSSION}

This study sought to evaluate current laboratory practices when confronted with suspected misattributed parentage (mainly misattributed paternity) encountered during familybased exome or genome sequencing. A majority of the clinical laboratories surveyed do not explicitly state anything about misattributed parentage in the report when found; however, laboratories do inform the clinicians about these findings. Laboratories overwhelmingly relied on the ordering clinician's preferences regarding the report, and therefore, what is typically reported varies greatly between laboratories and often within the same laboratory. It is difficult to construct a report that pleases those who do not wish to disclose misattributed parentage yet accurately states the laboratory's results for legal purposes. Our findings are comparable with those previously reported ${ }^{7}$ and show that most laboratory geneticists prefer not to state social incidental findings (such as familial relationships) on reports. However, knowing correct familial relationships is important for determining recurrence risks for couples as well as siblings. Thus, there are potentially clinically valid reasons for disclosing this information to clinicians and families, though there may still be specific clinical scenarios where this information should be left off of the written report.

\section{Consenting of patients/families}

The challenges in creating appropriate consent forms are notable; there is a delicate balance in keeping the readability manageable while acknowledging a number of potential complications that may arise. The ACMG position on informed consent for ES/GS includes recommendations that a medical geneticist or genetic counselor should counsel the patient/family, and this should include written documentation. ${ }^{9}$ The ACMG recommends that the patient/family should be informed of the potential consequences of familial testing as well as any testing alternatives, and the ASHG recommends that parents should be consented that misattributed parentage is a possibility. ${ }^{8}$ While the laboratories participating in this survey all have consent forms for ES/GS testing, not every laboratory necessarily requires a completed consent form prior to testing, and this survey did not specifically address whether laboratories would still proceed with testing for cases where documented consent was not obtained.

Regardless of the laboratory's decision on whether or not to disclose and how to do so, the consent form should ideally align with the action(s) the laboratory will take. Thus, a wellwritten consent should define the actions of the laboratory and alleviate the uncertainty of how to handle these situations for the laboratory personnel. This study, in agreement with 
others, ${ }^{10-12}$ found that consent forms are generally highly variable, from disclosing that information about misattributed parentage will be shared with the clinicians to not including any statement at all that would suggest misattributed parentage as a possible unanticipated finding. Most laboratories stated that "it may be detected" and nothing further, yet for all laboratories surveyed, they did indicate that they would inform the ordering clinician.

While one laboratory recently changed its consent to not be as explicit as before (so they are no longer routinely canceling tests where all submitted samples are not genetically related), another laboratory recently changed its consent form to include a more directed action that will be taken if misattributed parentage is encountered through NGS. That laboratory's new consent specifically states that they will be unable to fully interpret the results of the family-based test ordered, and therefore, misattributed parentage will be documented in the report as a possible explanation for the lack of genetic relatedness and this information will be shared with the ordering clinician. Because this could be perceived as forcing this information upon the patient's family, that laboratory also made more explicit the option to utilize alternative testing methods instead to achieve similar results (such as single-gene testing or proband-only NGS) in cases where there might be a question of biological parentage, which is in line with ACMG recommendations. This could then lead to an opportunity for the clinician or genetic counselor to have an open dialogue with the patient's family during the pretest counseling session about other testing methods, which would not include family-based ES/GS testing.

\section{Alternative testing without familial information}

Options for genetic testing vary in the quantity and/or types of unanticipated findings that may be encountered. Singlegene testing is most useful when the clinician is fairly certain of the diagnosis and mainly wants confirmation of a clinical suspicion. Gene panels allow for testing of multiple genes for syndromes where locus heterogeneity is higher. Both singlegene and gene panel testing generally only involve testing the proband. Some laboratories offer custom parental Sanger sequencing of variants found via proband-only exomes, gene panels, or single-gene testing to assist with classification; however, parental samples are generally only sequenced at that locus, and parentage is often not questioned or further assessed. However, this could conceivably lead to the misapplication of de novo evidence in variant classification.

Exome sequencing attempts to interrogate all coding genes in the genome and can be family based (trio or duo) or proband-only. Proband-only sequencing is typically performed for cases where the parents are unavailable, insurance coverage only allows for proband testing, the option aligns with the preferences of the ordering clinician/family/patient, or the suspected genetic etiology is unlikely to be informed by family-based testing (such as dominant inheritance). When exome sequencing costs were previously higher, many laboratories only offered singleton testing of the proband with or without assessment of candidate variants in the parental samples using Sanger sequencing. However, with the reduction in ES cost and acknowledgment of its importance in the medical field, family-based NGS testing has become more prevalent. Simultaneous assessment of parental samples helps to resolve variants based on inheritance; thus, there is a higher probability of finding the most appropriate causative variant(s). In addition, because the testing involves comparing variants against parental samples, unmatched parental samples are easier to determine. For example, while a matched exome or genome trio would be expected to only contain a few de novo variants in the proband, the observation of a greatly increased number of de novo variants in the proband should prompt further investigation by the laboratory, as it may be an indication that a genetically unrelated sample was used in the analysis.

\section{Laboratory reports}

Laboratory reporting language should be scientifically accurate and provide appropriate interpretation. In the case of a misattributed parentage, clinical laboratories must first decide if they are (or are not) going to provide written comments on the unrelated sample. Most clinical laboratories require some form of report for all samples successfully used in clinical testing; thus not reporting any results at all from a sample that comes into the laboratory is rarely done unless the quality of the specimen is determined to be poor (and this may then just be commented on within the laboratory information system). Another option would be to report that the sample was not used for a specific reason such as a genetic mismatch; for example, one could include a statement in the report with the number of Mendelian errors but not explicitly state anything about misattributed parentage. ${ }^{13}$ An additional option would be to report it as a general sample failure even though the nucleic acid quality was likely high and the sequencing data met all of the quality control specifications for an individual sample.

When misattributed parentage is suspected during familybased ES/GS, there are often several other possibilities besides true misattributed parentage that could result in similar findings, which laboratories may not be able to exclude and may want to report. These include a specimen mix-up during collection and handling or within the laboratory, sperm/egg donation, prior bone marrow engraftment in one of the presumptive parents, or incorrect family information provided to the laboratory. As a result, laboratories do not always have all of the necessary information to make formal conclusions regarding the true genetic relatedness of the submitted samples on a NGS report without gathering more information and/or performing further studies on repeat samples. Depending on the capabilities of the laboratory, a specimen mix-up at the point of intake (i.e., phlebotomist), delivery to the laboratory, and within the laboratory can be excluded by a second blood draw and comparison with the results from the previous sample (often using STRs). 
Laboratories must also decide how to report any variants found during testing. For example, if a parent carries a variant, yet does not phenotypically resemble the proband, the variant is often given less weight as being causative if it is in a gene associated with a dominant disease (adjusted for variable penetrance and expressivity). In a joint consensus statement, the ACMG and Association for Molecular Pathology (AMP) classify de novo as strong evidence of pathogenicity (PS2) when both maternity and paternity are confirmed, but it is only used as moderate evidence of pathogenicity when parentage for both parents is assumed but not confirmed (PM6). ${ }^{14}$ Thus, this highlights the importance of familial testing in regard to variant pathogenicity; however, if the laboratory has knowledge or suspicion of misattributed parentage, PM6 should not be applied. In a position statement, the ASHG also comments on the term de novo being used only when parental samples are confirmed. ${ }^{8}$ Many laboratories in this survey will comment on a variant not being inherited from only one comparator sample while failing to mention the other parental comparator; however, this reporting style may still lead to a discussion about misattributed parentage with the patient even if the second comparator was not specifically mentioned in the report.

\section{NGS versus STRs for genetic identity}

Forensic science and parentage testing typically use short tandem repeats (STRs) for analysis, which are repetitive sequences of DNA that vary in length among individuals (to create a variety of alleles). STR sizes are assessed and compared to identify related individuals or samples (for indications such as parentage testing, bone marrow engraftment, or maternal cell contamination). The more STRs with informative alleles that are tested, the higher the likelihood of identifying the correct result. For example, the Federal Bureau of Investigation (FBI) routinely uses 13 core loci for identification for forensic purposes. ${ }^{15}$

However, NGS is not typically utilized for parentage testing. While misattributed parentage may be suspected through genetic testing, these findings would currently be verified by formal STR testing even though NGS is far more informative than STR-based testing (or any other molecular method). As an example, "ultra-deep" NGS, unlike STR analysis, has been able to solve parentage and/or forensic cases in which monozygotic twins are involved due to rare variants that occur after the human blastocyst splits. ${ }^{16}$

While NGS may lead a clinical laboratory to suspect misattributed parentage after reviewing the data, clinical laboratories often avoid making that type of claim because the test ordered was not a parentage test. Parentage testing has strict rules in regard to chain of custody of samples. Chain of custody involves extensive documentation (e.g., identification documents, photographs) of the person giving their sample (i.e., the parents), how the specimen is obtained, and who touches the sample before it is tested. ${ }^{17}$ Extensive chain of custody documentation of the sample is not typically collected for many routine family-based exome and genome tests; therefore, laboratories do not often feel that they have all of the necessary information required to formally conclude misattributed parentage even if the NGS data appears convincing.

\section{Direct-to-consumer testing}

In the past several years, the direct-to-consumer (DTC) genetic testing industry has exploded in the general population. This fascination with personal genetics and genomics has increased genetic awareness; thus to assume that patients will not be able to deduce misattributed parentage from the results, even using genetic jargon, may be irresponsible. Interestingly, many of the DTC companies have little to no information in their policies and terms of service about unexpected findings such as misattributed parentage. ${ }^{18}$ However, if the family unit has DTC testing performed with the same company, many "reports" allow for genetic connections to be deduced using the results given to customers. ${ }^{19}$ While geneticists understand that DTC companies are not diagnostic laboratories and are not currently held to the same standard, ${ }^{20}$ the public has generally accepted them so far, and thus familial relationships will be questioned if the DTC results contradict previously held personal conceptions of familial relationships.

\section{Current limited professional guidance}

The ASHG recommends that health-care providers avoid disclosure of misattributed parentage unless a clear medical benefit outweighs the potential harm. However, the ASHG does not directly address the laboratory's role in disclosure on reports, only recommending that laboratory reports are detailed and accurate while facilitating comprehension by providers. ${ }^{8}$ Parentage testing is mentioned in a 2013 ACMG policy statement; however, the recommendation comments on mismatches at a single locus or chromosomal region being insufficient, ${ }^{21}$ not hundreds along the genome, which can be found through ES or GS. The ACMG and ASHG also have ES/GS consent recommendations; however, neither have issued recommendations for proper laboratory reporting of possible incorrect biological relationships. Therefore, professional recommendations may be warranted for the appropriate handling of suspected misattributed parentage encountered during family-based ES/GS to help standardize current clinical laboratory practices.

\section{ELECTRONIC SUPPLEMENTARY MATERIAL}

The online version of this article (https://doi.org/10.1038/s41436018-0265-4) contains supplementary material, which is available to authorized users.

\section{DISCLOSURE}

All authors are clinical service providers and are employed by laboratories that offer fee-based clinical sequencing. This employment is noted in the author affiliations. The authors declare no additional conflicts of interest beyond their employment affiliation. 


\section{REFERENCES}

1. Green RC, Berg JS, Grody WW, et al. ACMG recommendations for reporting of incidental findings in clinical exome and genome sequencing. Genet Med. 2013;15:565-574.

2. Kalia SS, Adelman K, Bale SJ, et al. Recommendations for reporting of secondary findings in clinical exome and genome sequencing, 2016 update (ACMG SF v2.0): a policy statement of the American College of Medical Genetics and Genomics. Genet Med. 2017;19:249-255.

3. Rehder CW, David KL, Hirsch B, Toriello HV, Wilson CM, Kearney HM. Response to Rosenberg et al. Genet Med. 2013;15:754.

4. Rehder CW, David KL, Hirsch B, Toriello HV, Wilson CM, Kearney HM. American College of Medical Genetics and Genomics: standards and guidelines for documenting suspected consanguinity as an incidental finding of genomic testing. Genet Med. 2013;15:150-152.

5. Protection of human subjects; reports of the President's Commission for the Study of Ethical Problems in Medicine and Biomedical and Behavioral Research-Office of the Assistant Secretary for Health, HHS. Notice of availability of reports. Fed Regist. 1983;48:34408-12.

6. Institute of Medicine Committee on Assessing Genetic Risks. In: Andrews LB, Fullarton JE, Holtzman NA, Motulsky AG, eds. The National Academies collection: reports funded by National Institutes of Health. Chapter 2: Genetic Testing and Assessment Washington, DC: National Academies Press; 1994:59-115.

7. Lohn Z, Adam S, Birch P, Townsend A, Friedman J. Genetics professionals' perspectives on reporting incidental findings from clinical genome-wide sequencing. Am J Med Genet A. 2013;161A:542-549.

8. Botkin JR, Belmont JW, Berg JS, et al. Points to consider: ethical, legal, and psychosocial implications of genetic testing in children and adolescents. Am J Hum Genet. 2015;97:6-21.

9. ACMG Board of Directors. Points to consider for informed consent for genome/exome sequencing. Genet Med. 2013;15:748-749.

10. Bergner $\mathrm{AL}$, Bollinger J, Raraigh $\mathrm{KS}$, et al. Informed consent for exome sequencing research in families with genetic disease: the emerging issue of incidental findings. Am J Med Genet A. 2014;164A:2745-2752.
11. Niemiec E, Vears DF, Borry $P$, Howard HC. Readability of informed consent forms for whole-exome and whole-genome sequencing. J Community Genet. 2018;9:143-151.

12. Fowler SA, Saunders CJ, Hoffman MA. Variation among consent forms for clinical whole exome sequencing. J Genet Couns. 2018;27:104-114.

13. Hehir-Kwa JY, Claustres M, Hastings RJ, et al. Towards a European consensus for reporting incidental findings during clinical NGS testing. Eur J Hum Genet. 2015;23:1601-1606.

14. Richards S, Aziz N, Bale S, et al. Standards and guidelines for the interpretation of sequence variants: a joint consensus recommendation of the American College of Medical Genetics and Genomics and the Association for Molecular Pathology. Genet Med. 2015;17:405-424.

15. Budowle B, Moretti TR, Baumstark AL, Defenbaugh DA, Keys KM. Population data on the thirteen CODIS core short tandem repeat loci in African Americans, U.S. Caucasians, Hispanics, Bahamians, Jamaicans, and Trinidadians. J Forensic Sci. 1999;44:1277-1286.

16. Weber-Lehmann J, Schilling E, Gradl G, Richter DC, Wiehler J, Rolf B. Finding the needle in the haystack: differentiating "identical" twins in paternity testing and forensics by ultra-deep next generation sequencing. Forensic Sci Int Genet. 2014;9:42-46.

17. Morling N, Allen R, Carracedo A, et al. Paternity Testing Commission of the International Society of Forensic Genetics. Recommendations on genetic investigations in paternity cases. Int J Leg Med. 2003;117:51-61.

18. Moray N, Pink KE, Borry $\mathrm{P}$, Larmuseau MH. Paternity testing under the cloak of recreational genetics. Eur J Hum Genet. 2017;25:768-770.

19. Kirkpatrick BE, Rashkin MD. Ancestry testing and the practice of genetic counseling. J Genet Couns. 2017;26:6-20.

20. Tandy-Connor S, Guiltinan J, Krempely K, et al. False-positive results released by direct-to-consumer genetic tests highlight the importance of clinical confirmation testing for appropriate patient care. Genet Med 2018 Mar 22; https://doi.org/10.1038/gim.2018.38. [Epub ahead of print].

21. Ross LF, Saal HM, David KL, Anderson RR, American Academy of Pediatrics, American College of Medical Genetics and Genomics. Technical report: Ethical and policy issues in genetic testing and screening of children. Genet Med. 2013;15:234-245. 